\title{
Wiener Index of Graphs and Their Line Graphs
}

\author{
Xiaohai Su • Ligong Wang · Yun Gao
}

Received: 9 May 2013 / Revised: 26 August 2013 / Accepted: 27 August 2013 /

Published online: 19 September 2013

(C) Operations Research Society of China, Periodicals Agency of Shanghai University, and

Springer-Verlag Berlin Heidelberg 2013

\begin{abstract}
The Wiener index $W(G)$ of a graph $G$ is a distance-based topological index defined as the sum of distances between all pairs of vertices in $G$. It is shown that for $\lambda=2$ there is an infinite family of planar bipartite chemical graphs $G$ of girth 4 with the cyclomatic number $\lambda$, but their line graphs are not chemical graphs, and for $\lambda \geqslant 2$ there are two infinite families of planar nonbipartite graphs $G$ of girth 3 with the cyclomatic number $\lambda$; the three classes of graphs have the property $W(G)=$ $W(L(G))$, where $L(G)$ is the line graph of $G$.
\end{abstract}

Keywords Wiener index · Line graph · Cyclomatic number

Mathematics Subject Classification (2000) $05 \mathrm{C} 12$

This work was supported by the National Natural Science Foundation of China (No. 11171273).

Electronic supplementary material The online version of this article

(doi:10.1007/s40305-013-0027-6) contains supplementary material, which is available to authorized users.

X. Su $\cdot$ Y. Gao

School of Mathematics and Computer Science, Shaanxi University of Technology, Hanzhong, Shaanxi 723001, P.R. China

X. Su

e-mail: susuxiaohai@163.com

Y. Gao

e-mail: gyenjoy@126.com

L. Wang (凶)

Department of Applied Mathematics, School of Science, Northwestern Polytechnical University, Xi'an, Shaanxi 710072, P.R. China

e-mail: lgwangmath@163.com 


\section{Introduction}

All graphs considered in this paper are finite, undirected, connected, without loops and multiple edges. The vertex and edge sets of $G$ are denoted by $V(G)$ and $E(G)$, respectively. Let $n(G)$ denote the number of vertices in a graph $G$. The cyclomatic number $\lambda$ of a graph $G$ is defined as $\lambda(G)=|E(G)|-|V(G)|+1$. The line graph $L(G)$ of a graph $G$ has the vertex set $V(L(G))=E(G)$, and two distinct vertices of the graph $L(G)$ are adjacent if the corresponding edges of $G$ have a common endvertex. A chemical graph $G$ is a graph in which no vertex has degree greater than four. If $u$ and $v$ are vertices of $G$, then the number of edges in the shortest path connecting them is said to be their distance and is denoted by $d(u, v)$. The sum of distances from a vertex $v$ to all vertices in a graph $G$ is called the distance of this vertex, $d_{G}(v)=\sum_{u \in V(G)} d(u, v)$.

The Wiener index is a well-known distance-based topological index introduced as structural descriptor for acyclic organic molecules [17]. It is defined as the sum of distances between all unordered pairs of vertices of a simple graph $G$, i.e., $W(G)=$ $\sum_{\{u, v\} \subseteq V(G)} d(u, v)=\frac{1}{2} \sum_{v \in V(G)} d_{G}(v)$. For a graph $G$, let $\Delta W(G)=W(L(G))-$ $W(G)$.

Mathematical properties and chemical applications of the Wiener index have been intensively studied in the last 35 years. Nowadays, the Wiener index is one of the best understood and most frequently used molecular shape descriptors. It has found many applications in the modelling of physico-chemical, pharmacological and biological properties of organic molecules. For detailed information on the Wiener index and its applications, we refer to $[5,6,8,16]$. There are two groups of closely related problems which have attracted the attention of researchers for a long time: (a) how the Wiener index depends on the structure of a graph, (b) how the Wiener index changes under graph operations.

One of the interesting approaches in mathematical chemistry studies is to characterize molecular graphs by means of parameters calculated for their derived structures. The line graph can reflect branchings of the initial graph, it is a good example of derived structures of molecular graphs. Invariants of line graphs have been applied for the evaluation of structural complexity of molecular graphs, for ordering of structures and for the design of novel topological indices [1, 12, 14].

In this paper, we are interested in finding graphs of girth $g$ satisfying the following equality:

$$
\Delta W(G)=0 \quad(\text { i.e. } W(L(G))=W(G))
$$

and having prescribed cyclomatic number $\lambda(G)$. It was shown that the Wiener index of a tree $(\lambda(G)=0)$ and its line graph are always distinct [2]. For any unicyclic graph $G(\lambda(G)=1)$, except the simple cycle, $W(L(G))<W(G)$ [13]. Therefore, if a cycle-containing graph, except a simple cycle, satisfies the equality (1.1), then it has at least two cycles $(\lambda(G)=2)[13,15]$. There are exactly 26 minimal bicyclic graphs of order 9 having property (1.1) [7]. The numbers of bicyclic graphs of order 10, 11 and 12 satisfying (1.1) are 166, 503 and 1082, respectively [7]. Series of bicyclic graphs with increasing order have been constructed in [15]. Minimal tricyclic graphs $(\lambda(G)=3$ ) with property (1.1) have 12 vertices (71 graphs) [7]. Two infinite 
Fig. $1 G_{r, t}$

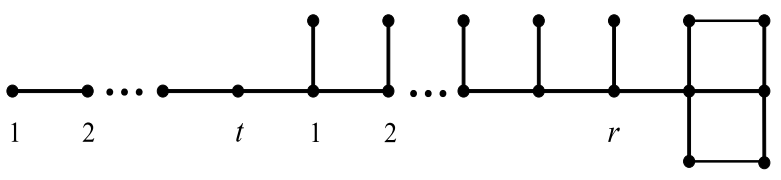

Fig. 2 The line graph $L\left(G_{r, t}\right)$ of $G_{r, t}$

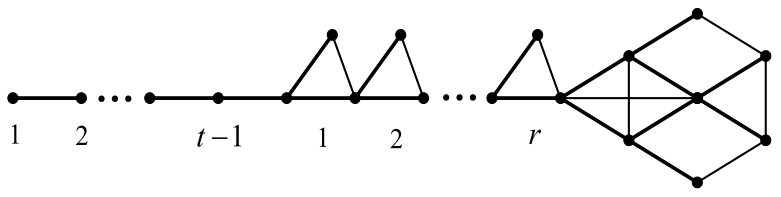

families of planar nonbipartite graphs of girth 3 or girth 4 with increasing cyclomatic number having property (1.1) are constructed in [9]. Dobrynin and Mel'nikov in $[10,11]$ proved that for every $\lambda(G) \geqslant 3$ there are planar bipartite graphs $G$ of girth 4 with cyclomatic number $\lambda(G)$ having property (1.1). Cohen et al. in [3] proved that for every nonnegative integer $g_{0}$, there exists $g>g_{0}$, such that there are planar nonbipartite graphs $G$ of girth $g$ with cyclomatic number $\lambda(G)=2$ having property (1.1). Deng [4] constructed an infinite family of planar bipartite chemical graphs $G$ of girth 4 with cyclomatic number $\lambda(G)=2$ having property (1.1), and their line graphs are also chemical graphs. In this paper, we construct an infinite family of planar bipartite chemical graphs $G$ of girth 4 with cyclomatic number $\lambda(G)=2$ having property (1.1), but their line graphs are not chemical graphs. We also obtain two families of planar nonbipartite graphs $G$ of girth 3 with increasing cyclomatic numbers having property (1.1).

\section{Main Results}

We introduce the following useful lemma for computing the Wiener index of a graph.

Lemma $2.1([6,10])$ Let $G$ be the graph obtained by identifying a vertex $u$ of $G_{1}$ and $a$ vertex $v$ of $G_{2}$. Then

$$
W(G)=W\left(G_{1}\right)+W\left(G_{2}\right)+\left(n\left(G_{1}\right)-1\right) d_{G_{2}}(v)+\left(n\left(G_{2}\right)-1\right) d_{G_{1}}(u) .
$$

Firstly, we shall construct an infinite family of planar bipartite chemical graphs of girth 4 with the cyclomatic number $\lambda=2$ having property (1.1). Consider the graph $G_{r, t}$ shown in Fig. 1. By construction, it has cyclomatic number $\lambda=2$ and order $2 r+t+6$. $G_{r, t}$ is a planar and bipartite graph for every $r$ and $t$. The structure of the line graph $L\left(G_{r, t}\right)$ is depicted in Fig. 2.

Lemma 2.2 For positive integers $r$ and $t$, let $G_{r, t}$ be the graph of girth 4 shown in Fig. 1, then $\Delta W\left(G_{r, t}\right)=-3 r-\frac{5}{2} t+\frac{1}{2} t^{2}+8$. 


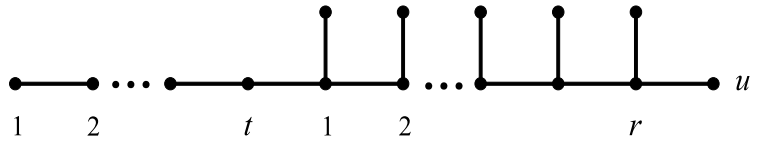

T

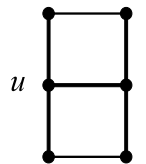

$G_{1}$

Fig. 3 The subgraphs $T$ and $G_{1}$ of $G_{r, t}$
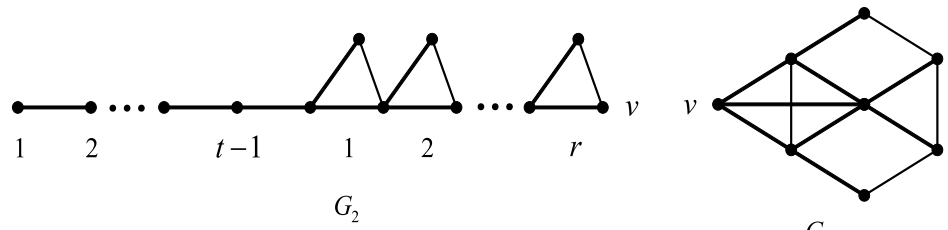

$G_{3}$

Fig. 4 The subgraphs $G_{2}$ and $G_{3}$ of line graph $L\left(G_{r, t}\right)$

Proof For the tree $T$ shown in Fig. 3, by Lemma 2.1 or [4] and the definition of $d_{T}(u)$, we have

$$
\begin{aligned}
W(T) & =\frac{1}{3} r+3 r^{2}+\frac{2}{3} r^{3}+\frac{1}{3} t+2 r t+r^{2} t+\frac{1}{2} t^{2}+r t^{2}+\frac{1}{6} t^{3}, \\
n(T) & =2 r+t+1,
\end{aligned}
$$

and $d_{T}(u)=r^{2}+3 r+1+(r+1)(t-1)+\frac{1}{2} t(t-1)$.

For the graph $G_{1}$ shown in Fig. 3, we have $W\left(G_{1}\right)=25, n\left(G_{1}\right)=6, d_{G_{1}}(u)=7$, hence by Lemma 2.1, we have

$$
\begin{aligned}
W\left(G_{r, t}\right)= & W(T)+W\left(G_{1}\right)+7(2 r+t) \\
& +5\left(r^{2}+3 r+1+(r+1)(t-1)+\frac{1}{2} t(t-1)\right) \\
= & \frac{1}{3} r+3 r^{2}+\frac{2}{3} r^{3}+\frac{1}{3} t+2 r t+r^{2} t+\frac{1}{2} t^{2}+r t^{2}+\frac{1}{6} t^{3}+25+14 r+7 t \\
& +5 r^{2}+15 r+5+5 r t-5 r+5 t-5+\frac{5}{2} t^{2}-\frac{5}{2} t \\
& \frac{73}{3} r+8 r^{2}+\frac{2}{3} r^{3}+\frac{59}{6} t+7 r t+r^{2} t+3 t^{2}+r t^{2}+\frac{1}{6} t^{3}+25 .
\end{aligned}
$$

For the graphs $G_{2}$ and $G_{3}$ shown in Fig. 4, we have

$$
\begin{aligned}
& W\left(G_{2}\right)=-\frac{2}{3} r+r^{2}+\frac{2}{3} r^{3}-\frac{1}{6} t+r^{2} t+r t^{2}+\frac{1}{6} t^{3}, \quad n\left(G_{2}\right)=2 r+t, \\
& d_{G_{2}}(v)=r(r+1)+r(t-1)+\frac{1}{2} t(t-1)=r^{2}+r t+\frac{1}{2} t^{2}-\frac{1}{2} t, \\
& W\left(G_{3}\right)=44, \quad n\left(G_{3}\right)=8, \quad d_{G_{3}}(v)=11 .
\end{aligned}
$$



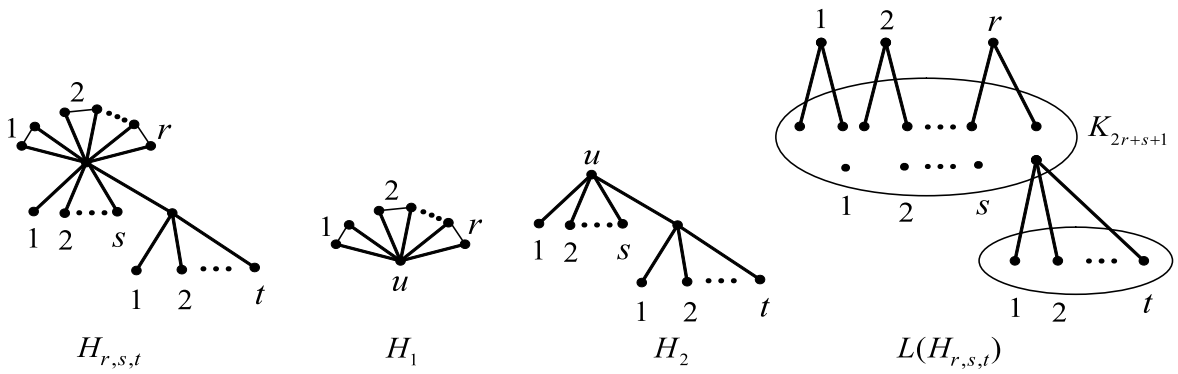

Fig. $5 H_{r, s, t}$, the subgraphs $H_{1}$ and $H_{2}$ of $H_{r, s, t}$, the line graph $L\left(H_{r, s, t}\right)$ of $H_{r, s, t}$

By Lemma 2.1, we have

$$
\begin{aligned}
W\left(L\left(G_{r, t}\right)\right)= & 44-\frac{2}{3} r+r^{2}+\frac{2}{3} r^{3}-\frac{1}{6} t+r^{2} t+r t^{2}+\frac{1}{6} t^{3}+11(2 r+t-1) \\
& +7\left(r^{2}+r t+\frac{1}{2} t^{2}-\frac{1}{2} t\right) \\
= & \frac{64}{3} r+8 r^{2}+\frac{2}{3} r^{3}+\frac{44}{6} t+7 r t+r^{2} t+\frac{7}{2} t^{2}+r t^{2}+\frac{1}{6} t^{3}+33 .
\end{aligned}
$$

Hence, we have $\Delta W\left(G_{r, t}\right)=W\left(L\left(G_{r, t}\right)\right)-W\left(G_{r, t}\right)=-3 r+\frac{1}{2} t^{2}-\frac{5}{2} t+8$.

Theorem 2.3 There is an infinite family of planar bipartite chemical graphs $G_{r, t}$ of girth 4 with cyclomatic number $\lambda\left(G_{r, t}\right)=2$ such that $\Delta W\left(G_{r, t}\right)=0$. Specifically, $\Delta W\left(G_{r, t}\right)=0$ if and only if $t=3 k-2, r=\left(3 k^{2}-9 k+10\right) / 2$, where $k$ is any positive integer.

Proof From Lemma 2.2, we know that $\Delta W\left(G_{r, t}\right)=0$ if and only if $-3 r+\frac{1}{2} t^{2}-\frac{5}{2} t+$ $8=0$. Hence, all of the positive solutions of this equation are given by $t=3 k-2$, $r=\left(3 k^{2}-9 k+10\right) / 2=3 k(k-3) / 2+5$, where $k$ is any positive integer. Since one of two integers $k$ and $k-3$ must be even, hence $r$ is a positive integer. The proof of Theorem 2.3 is completed.

Secondly, we shall construct an infinite family of planar nonbipartite graphs of girth 3 with increasing cyclomatic number having property (1.1). For nonnegative integers $r(\geqslant 1), s$ and $t$, consider the graph $H_{r, s, t}$ shown in Fig. 5. By construction, it has cyclomatic number $\lambda=r$ and order $2 r+s+t+2$. $H_{r, s, t}$ is a planar nonbipartite graph for every $r, s$ and $t$. The structure of the line graph $L\left(H_{r, s, t}\right)$ is depicted in Fig. 5, its complete subgraphs are drawn without edges.

Lemma 2.4 For nonnegative integers $r(\geqslant 1), s$ and $t$, let $H_{r, s, t}$ be the graph of girth 3 shown in Fig. 5. Then $\Delta W\left(H_{r, s, t}\right)=\frac{7}{2} r^{2}-\frac{1}{2} s^{2}-\frac{1}{2} t^{2}-s t-\frac{3}{2} s-\frac{3}{2} t-\frac{7}{2} r+r t-1$.

Proof Since $W\left(H_{1}\right)=4 r^{2}-r, d_{H_{1}}(u)=2 r, n\left(H_{1}\right)=2 r+1$ and

$$
W\left(H_{2}\right)=s^{2}+t^{2}+3 s t+2 s+2 t+1, \quad d_{H_{2}}(u)=s+2 t+1, \quad n\left(H_{2}\right)=s+t+2 .
$$


By Lemma 2.1, we have

$$
W\left(H_{r, s, t}\right)=4 r^{2}+s^{2}+t^{2}+3 s t+2 s+2 t+3 r+4 r s+6 r t+1 .
$$

The Wiener index of the line graph $L\left(H_{r, s, t}\right)$ can be easily calculated:

$$
W\left(L\left(H_{r, s, t}\right)\right)=\frac{15}{2} r^{2}+\frac{1}{2} s^{2}+\frac{1}{2} t^{2}+2 s t+\frac{1}{2} s+\frac{1}{2} t-\frac{1}{2} r+4 r s+7 r t .
$$

Hence, we have

$$
\begin{aligned}
\Delta W\left(H_{r, s, t}\right) & =W\left(L\left(H_{r, s, t}\right)\right)-W\left(H_{r, s, t}\right) \\
& =\frac{7}{2} r^{2}-\frac{1}{2} s^{2}-\frac{1}{2} t^{2}-s t-\frac{3}{2} s-\frac{3}{2} t-\frac{7}{2} r+r t-1 .
\end{aligned}
$$

Theorem 2.5 For every integer $\lambda \geqslant 2$, there is an infinite family of planar nonbipartite graphs $H_{r, s, t}$ of girth 3 with the cyclomatic number $\lambda=r$ such that $\Delta W\left(H_{r, s, t}\right)=0$. Specifically, $\Delta W\left(H_{r, s, t}\right)=0$ if and only if (i) $s=2 r-2 k-4-$ $k(k-1) /(2 r) \geqslant 0, t=r+3 k+2+k(k-1) /(2 r) \geqslant 0$, or (ii) $s=2 r+2 k-6-$ $k(k-1) /(2 r) \geqslant 0, t=r-3 k+5+k(k-1) /(2 r) \geqslant 0$, where $r(\geqslant 2)$ and $k(\geqslant 0)$ are integers, and $k(k-1) /(2 r)$ must be a nonnegative integer.

Proof From Lemma 2.4, let $\Delta W\left(H_{r, s, t}\right)=0$, then

$$
\frac{7}{2} r^{2}-\frac{1}{2} s^{2}-\frac{1}{2} t^{2}-s t-\frac{3}{2} s-\frac{3}{2} t-\frac{7}{2} r+r t-1=0 .
$$

Multiply two sides of the above equation by 8 and complete the square on the above equation such that its left side becomes the difference of two forms of squares, we immediately obtain the following equation:

$$
(2 s+2 t+3)^{2}-(6 r)^{2}=-8 r^{2}+8 r t-28 r+1 .
$$

Let $x=2 s+2 t+3, y=6 r, a=-8 r^{2}+8 r t-28 r+1$, then we have

$$
x^{2}-y^{2}=a .
$$

From a result in elementary number theory, we know that the Diophantine equation (2.2) has solutions if and only if $2 \nmid a$ or $4 \mid a$.

Since $a=-8 r^{2}+8 r t-28 r+1$ is odd, we only consider the case that both $x+y$ and $x-y$ are odds. Then, for any nonnegative integer $k$, we take

$$
\left\{\begin{array} { l } 
{ x + y = \frac { - 8 r ^ { 2 } + 8 r t - 2 8 r + 1 } { 2 k - 1 } , } \\
{ x - y = 2 k - 1 , }
\end{array} \text { or } \quad \left\{\begin{array}{l}
x+y=-\frac{-8 r^{2}+8 r t-28 r+1}{2 k-1}, \\
y-x=2 k-1,
\end{array}\right.\right.
$$

where $\left(-8 r^{2}+8 r t-28 r+1\right) /(2 k-1)$ must be an integer.

Then we have

$$
\left\{\begin{array} { l } 
{ x = \frac { - 4 r ^ { 2 } + 4 r t - 1 4 r + 2 k ^ { 2 } - 2 k + 1 } { 2 k - 1 } , } \\
{ y = \frac { - 4 r ^ { 2 } + 4 r t - 1 4 r - 2 k ^ { 2 } + 2 k } { 2 k - 1 } , }
\end{array} \text { or } \quad \left\{\begin{array}{l}
x=-\frac{-4 r^{2}+4 r t-14 r+2 k^{2}-2 k+1}{2 k-1}, \\
y=-\frac{-4 r^{2}+4 r t-14 r-2 k^{2}+2 k}{2 k-1} .
\end{array}\right.\right.
$$


Thus,

$$
\left\{\begin{array} { l } 
{ s = 2 r - 2 k - 4 - \frac { k ( k - 1 ) } { 2 r } \geqslant 0 , } \\
{ t = r + 3 k + 2 + \frac { k ( k - 1 ) } { 2 r } \geqslant 0 , }
\end{array} \quad \text { or } \quad \left\{\begin{array}{l}
s=2 r+2 k-6-\frac{k(k-1)}{2 r} \geqslant 0, \\
t=r-3 k+5+\frac{k(k-1)}{2 r} \geqslant 0,
\end{array}\right.\right.
$$

where $r(\geqslant 1)$ and $k(\geqslant 0)$ are integers, and $k(k-1) /(2 r)$ must be an integer.

Hence, $\Delta W\left(H_{r, s, t}\right)=0$ if and only if (i) $s=2 r-2 k-4-k(k-1) /(2 r) \geqslant 0$, $t=r+3 k+2+k(k-1) /(2 r) \geqslant 0$, or (ii) $s=2 r+2 k-6-k(k-1) /(2 r) \geqslant 0$, $t=r-3 k+5+k(k-1) /(2 r) \geqslant 0$, where $r(\geqslant 1)$ and $k(\geqslant 0)$ are integers, and $k(k-1) /(2 r)$ must be a nonnegative integer. Therefore, for every integer $\lambda=r \geqslant 2$, there is an infinite family of planar nonbipartite graphs $H_{r, s, t}$ of girth 3 with the cyclomatic number $\lambda$ such that $W\left(H_{r, s, t}\right)=W\left(L\left(H_{r, s, t}\right)\right)$. The proof of Theorem 2.5 is completed.

Based on Theorem 2.5 we obtain the following.

Corollary 2.6 Let $r, s, t$ and $k$ be as in Theorem 2.5, and let $r(\geqslant 1), s(\geqslant 0), t(\geqslant 0)$ and $k(\geqslant 0)$ be integers. Then the graph $H_{r, s, t}$ of girth 3 with the cyclomatic number $\lambda=r$ satisfies the equality $\Delta W\left(H_{r, s, t}\right)=0$ if one of the following cases holds where $l(\geqslant 1)$ and $m(\geqslant 0)$ are integers, $k, r, s, t$ in (1)-(11) are as in Theorem 2.5(i), and $k$, $r, s, t$ in (12)-(20) are as in Theorem 2.5(ii).

(1) $k=0, r=l, s=2 l-4, t=l+2$, where $l \geqslant 2$.

(2) $k=1, r=l, s=2 l-6, t=l+5$, where $l \geqslant 3$.

(3) $k=l, r=l(l-1) / 2, s=l^{2}-3 l-5, t=(l+2)(l+3) / 2$, where $l \geqslant 5$.

(4) $k=2 l m, r=l(2 l m-1), s=4 l^{2} m-4 l m-2 l-m-4, t=2 l^{2} m+6 l m-l+$ $m+2$, where $l=2, m \geqslant 2$ or $l \geqslant 3, m \geqslant 1$.

(5) $k=2 l m+1, r=l(2 l m+1), s=4 l^{2} m-4 l m+2 l-m-6, t=2 l^{2} m+6 l m+$ $l+m+5$, where $l=2, m \geqslant 1$ or $l \geqslant 3, m \geqslant 0$.

(6) $k=2(2 l m+l+m+1), r=(2 l+1)(2 l m+l+m+1), s=2(2 l-1)(2 l m+$ $l+m+1)-2 m-5, t=(2 l+7)(2 l m+l+m+1)+2 m+3$, where $l=1$, $m \geqslant 1$ or $l \geqslant 2, m \geqslant 0$.

(7) $k=(2 l+1)(2 m+1), r=(2 l+1)(2 l m+l+m), s=2(2 l+1)(2 l m+l-m-$ $1)-2 m-5, t=(2 l+1)(2 l m+l+7 m+3)+2 m+3$, where $l=1, m \geqslant 2$ or $l \geqslant 2, m \geqslant 2$.

(8) $k=2 m(2 l+1), r=(2 l+1)(4 l m+2 m-1), s=2(2 l+1)(4 l m-1)-m-4$, $t=(2 l+1)(4 l m+8 m-1)+m+2$, where $l \geqslant 1, m \geqslant 1$.

(9) $k=(4 l-1)(4 m-1), r=(4 l-1)(4 l m-l-m), s=2(4 l-1)(4 l m-l-5 m+$ 1) $-8 m-2, t=(4 l-1)(4 l m-l+11 m-3)+8 m$, where $l \geqslant 2, m \geqslant 1$.

(10) $k=(8 l-5)(8 m-5), r=(8 l-5)(8 l m-5 l-5 m+3), s=2(8 l-5)(8 l m-$ $5 l-13 m+8)-32 m+16, t=(8 l-5)(8 l m-5 l+19 m-12)+32 m-18$, where $l \geqslant 2, m \geqslant 1$.

(11) $k=4(4 l m-l+m), r=(4 l+1)(4 l m-l+m), s=2(4 l-3)(4 l m-l+m)-$ $8 m-2, t=(4 l+13)(4 l m-l+m)+8 m$, where $l=1, m \geqslant 2$ or $l \geqslant 2, m \geqslant 1$.

(12) $k=l, r=l(l-1) / 2, s=l^{2}+l-7, t=(l-3)(l-4) / 2$, where $l \geqslant 3$.

(13) $k=2 l m, r=l(2 l m-1), s=4 l^{2} m+4 l m-2 l-m-6, t=2 l^{2} m-6 l m-l+$ $m+5$, where $l=2, m=1$ or $l \geqslant 3, m \geqslant 1$. 


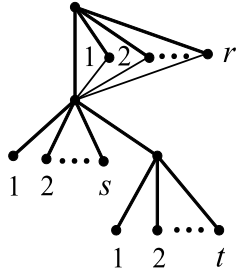

$R_{r, s, t}$

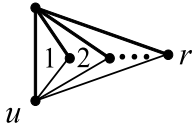

$R_{1}$

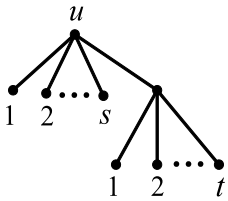

$R_{2}$

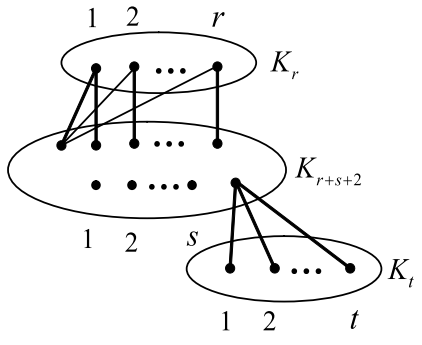

$L\left(R_{r, s, t}\right)$

Fig. $6 R_{r, s, t}$, the subgraphs $R_{1}$ and $R_{2}$ of $R_{r, s, t}$, the line graph $L\left(R_{r, s, t}\right)$ of $R_{r, s, t}$

(14) $k=2 l m+1, r=l(2 l m+1), s=4 l^{2} m+4 l m+2 l-m-4, t=2 l^{2} m-6 l m+$ $l+m+2$, where $l=1, m=1 ; l=2, m=0 ; l=2, m=1$ or $l \geqslant 3, m \geqslant 0$.

(15) $k=2(2 l m+l+m+1), r=(2 l+1)(2 l m+l+m+1), s=2(2 l+3)(2 l m+$ $l+m+1)-2 m-7, t=(2 l-5)(2 l m+l+m+1)+2 m+6$, where $l=1$, $m=0 ; l=2, m=0 ; l=2, m=1$ or $l \geqslant 3, m \geqslant 0$.

(16) $k=(2 l+1)(2 m+1), r=(2 l+1)(2 l m+l+m), s=2(2 l+1)(2 l m+l+3 m+$ 1) $-2 m-7, t=(2 l+1)(2 l m+l-5 m-3)+2 m+6$, where $l=1, m=0$; $l=2, m=0$ or $l \geqslant 3, m \geqslant 0$.

(17) $k=2 m(2 l+1), r=(2 l+1)(4 l m+2 m-1), s=2(2 l+1)(4 l m+4 m-1)-$ $m-6, t=(2 l+1)(4 l m-4 m-1)+m+5$, where $l \geqslant 1, m \geqslant 1$.

(18) $k=(4 l-1)(4 m-1), r=(4 l-1)(4 l m-l-m), s=2(4 l-1)(4 l m-l+3 m-$ 1) $-8 m-4, t=(4 l-1)(4 l m-l-13 m+3)+8 m+3$, where $l \geqslant 4, m \geqslant 1$.

(19) $k=(8 l-5)(8 m-5), r=(8 l-5)(8 l m-5 l-5 m+3), s=2(8 l-5)(8 l m-$ $5 l+3 m-2)-32 m+14, t=(8 l-5)(8 l m-5 l-29 m+18)+32 m-15$, where $l \geqslant 4, m \geqslant 1$.

(20) $k=4(4 l m-l+m), r=(4 l+1)(4 l m-l+m), s=2(4 l+5)(4 l m-l+m)-$ $8 m-4, t=(4 l-11)(4 l m-l+m)+8 m+3$, where $l \geqslant 3, m \geqslant 1$.

Thirdly, we shall construct another infinite family of planar nonbipartite graphs of girth 3 with increasing cyclomatic number having property (1.1). For nonnegative integers $r(\geqslant 1), s$ and $t$, consider the graph $R_{r, s, t}$ shown in Fig. 6. By construction, it has cyclomatic number $\lambda=r$ and order $r+s+t+3$. $R_{r, s, t}$ is a planar nonbipartite graph for every $r, s$ and $t$. The structure of the line graph $L\left(R_{r, s, t}\right)$ is depicted in Fig. 6, its complete subgraphs are drawn without edges.

Lemma 2.7 For nonnegative integers $r(\geqslant 1), s$ and $t$, let $R_{r, s, t}$ be the graph of girth 3 shown in Fig. 6. Then $\Delta W\left(R_{r, s, t}\right)=2 r^{2}-\frac{1}{2} s^{2}-\frac{1}{2} t^{2}-s t+r s+2 r t-\frac{5}{2} s-\frac{5}{2} t-3$.

Proof Since $W\left(R_{1}\right)=r^{2}+r+1, d_{R_{1}}(u)=r+1, n\left(R_{1}\right)=r+2$ and $W\left(R_{2}\right)=$ $s^{2}+t^{2}+3 s t+2 s+2 t+1, d_{R_{2}}(u)=s+2 t+1, n\left(R_{2}\right)=s+t+2$, by Lemma 2.1, we have

$$
W\left(R_{r, s, t}\right)=r^{2}+s^{2}+t^{2}+3 s t+3 r t+2 r s+3 r+4 s+5 t+4 .
$$


The Wiener index of the line graph $L\left(R_{r, s, t}\right)$ can be easily calculated:

$$
W\left(L\left(R_{r, s, t}\right)\right)=3 r^{2}+\frac{1}{2} s^{2}+\frac{1}{2} t^{2}+2 s t+\frac{3}{2} s+\frac{5}{2} t+3 r+3 r s+5 r t+1 .
$$

Hence, we have $\Delta W\left(R_{r, s, t}\right)=2 r^{2}-\frac{1}{2} s^{2}-\frac{1}{2} t^{2}-s t+r s+2 r t-\frac{5}{2} s-\frac{5}{2} t-3$.

Theorem 2.8 For every integer $\lambda \geqslant 2$, there is an infinite family of planar nonbipartite graphs $R_{r, s, t}$ of girth 3 with the cyclomatic number $\lambda=r$ such that $\Delta W\left(R_{r, s, t}\right)=$ 0 . Specifically, $\Delta W\left(R_{r, s, t}\right)=0$ if and only if (i) $s=2 r-2 k-4-k(k-1) /(2 r) \geqslant 0$, $t=2 r+3 k+1+k(k-1) /(2 r) \geqslant 0$, or (ii) $s=2 r+2 k-6-k(k-1) /(2 r) \geqslant 0$, $t=2 r-3 k+4+k(k-1) /(2 r) \geqslant 0$, where $r(\geqslant 2)$ and $k(\geqslant 0)$ are integers, and $k(k-1) /(2 r)$ must be a nonnegative integer.

Proof From Lemma 2.7, let $\Delta W\left(R_{r, s, t}\right)=0$, then we obtain

$$
(2 s+2 t-2 r+5)^{2}-(6 r)^{2}=-16 r^{2}+8 r t-20 r+1 .
$$

Hence the result follows by using a similar method as in Theorem 2.5.

Based on Theorem 2.8 we obtain the following.

Corollary 2.9 Let $r, s, t$ and $k$ be as in Theorem 2.8, and let $r(\geqslant 1), s(\geqslant 0), t(\geqslant 0)$ and $k(\geqslant 0)$ be integers. Then the graph $R_{r, s, t}$ of girth 3 with the cyclomatic number $\lambda=r$ satisfies the equality $\Delta W\left(R_{r, s, t}\right)=0$ if one of the following cases holds where $l(\geqslant 1)$ and $m(\geqslant 0)$ are integers, $k, r, s, t$ in (1)-(11) are as in Theorem 2.8(i), and $k$, $r, s, t$ in (12)-(20) are as in Theorem 2.8(ii).

(1) $k=0, r=l, s=2 l-4, t=2 l+1$, where $l \geqslant 2$.

(2) $k=1, r=l, s=2 l-6, t=2 l+4$, where $l \geqslant 3$.

(3) $k=l, r=l(l-1) / 2, s=l^{2}-3 l-5, t=l^{2}+2 l+2$, where $l \geqslant 5$.

(4) $k=2 l m, r=l(2 l m-1), s=4 l^{2} m-4 l m-2 l-m-4, t=4 l^{2} m+6 l m-2 l+$ $m+1$, where $l=2, m \geqslant 2$ or $l \geqslant 3, m \geqslant 1$.

(5) $k=2 l m+1, r=l(2 l m+1), s=4 l^{2} m-4 l m+2 l-m-6, t=4 l^{2} m+6 l m+$ $2 l+m+4$, where $l=2, m \geqslant 1$ or $l \geqslant 3, m \geqslant 0$.

(6) $k=2(2 l m+l+m+1), r=(2 l+1)(2 l m+l+m+1), s=2(2 l-1)(2 l m+$ $l+m+1)-2 m-5, t=4(l+2)(2 l m+l+m+1)+2 m+2$, where $l=1$, $m \geqslant 1$ or $l \geqslant 2, m \geqslant 0$.

(7) $k=(2 l+1)(2 m+1), r=(2 l+1)(2 l m+l+m), s=2(2 l+1)(2 l m+l-m-$ $1)-2 m-5, t=(2 l+1)(4 l m+2 l+8 m+3)+2 m+2$, where $l=1, m \geqslant 2$ or $l \geqslant 2, m \geqslant 0$.

(8) $k=2 m(2 l+1), r=(2 l+1)(4 l m+2 m-1), s=2(2 l+1)(4 l m-1)-m-4$, $t=2(2 l+1)(4 l m+5 m-1)+m+1$, where $l \geqslant 1, m \geqslant 1$.

(9) $k=(4 l-1)(4 m-1), r=(4 l-1)(4 l m-l-m), s=2(4 l-1)(4 l m-l-5 m+$ 1) $-8 m-2, t=(4 l-1)(8 l m-2 l+10 m-3)+8 m-1$, where $l \geqslant 3, m \geqslant 1$.

(10) $k=(8 l-5)(8 m-5), r=(8 l-5)(8 l m-5 l-5 m+3), s=2(8 l-5)(8 l m-$ $5 l-13 m+8)-32 m+16, t=(8 l-5)(8 l m-10 l+14 m-9)+32 m-19$, where $l \geqslant 2, m \geqslant 1$. 
(11) $k=4(4 l m-l+m), r=(4 l+1)(4 l m-l+m), s=2(4 l-3)(4 l m-l+m)-$ $8 m-2, t=2(4 l+7)(4 l m-l+m)+8 m-1$, where $l=1, m \geqslant 2$ or $l \geqslant 2$, $m \geqslant 1$.

(12) $k=l, r=l(l-1) / 2, s=l^{2}+l-7, t=l^{2}-4 l+5$, where $l \geqslant 3$.

(13) $k=2 l m, r=l(2 l m-1), s=4 l^{2} m+4 l m-2 l-m-6, t=4 l^{2} m-6 l m-2 l+$ $m+4$, where $l=1, m=2$ or $l \geqslant 2, m \geqslant 1$.

(14) $k=2 l m+1, r=l(2 l m+1), s=4 l^{2} m+4 l m+2 l-m-4, t=4 l^{2} m-6 l m+$ $2 l+m+1$, where $l=1, m=1 ; l=1, m=2 ; l=1, m=3$ or $l \geqslant 2, m \geqslant 0$.

(15) $k=2(2 l m+l+m+1), r=(2 l+1)(2 l m+l+m+1), s=2(2 l+3)(2 l m+$ $l+m+1)-2 m-7, t=4(l-1)(2 l m+l+m+1)+2 m+5$, where $l \geqslant 1$, $m \geqslant 0$.

(16) $k=(2 l+1)(2 m+1), r=(2 l+1)(2 l m+l+m), s=2(2 l+1)(2 l m+l+3 m+$ 1) $-2 m-7, t=(2 l+1)(4 l m+2 l-4 m-3)+2 m+5$, where $l \geqslant 1, m \geqslant 0$.

(17) $k=2 m(2 l+1), r=(2 l+1)(4 l m+2 m-1), s=2(2 l+1)(4 l m+4 m-1)-$ $m-6, t=2(2 l+1)(4 l m-m-1)+m+4$, where $l \geqslant 1, m \geqslant 1$.

(18) $k=(4 l-1)(4 m-1), r=(4 l-1)(4 l m-l-m), s=2(4 l-1)(4 l m-l+3 m-$ $1)-8 m-4, t=(4 l-1)(8 l m-2 l-14 m+3)+8 m+2$, where $l \geqslant 2, m \geqslant 1$.

(19) $k=(8 l-5)(8 m-5), r=(8 l-5)(8 l m-5 l-5 m+3), s=2(8 l-5)(8 l m-$ $5 l+3 m-2)-32 m+14, t=(8 l-5)(16 l m-10 l-34 m+21)+32 m-16$, where $l \geqslant 2, m \geqslant 1$.

(20) $k=4(4 l m-l+m), r=(4 l+1)(4 l m-l+m), s=2(4 l+5)(4 l m-l+m)-$ $8 m-4, t=2(4 l-5)(4 l m-l+m)+8 m+2$, where $l=1, m=1 ; l=1, m=2$ or $l \geqslant 2, m \geqslant 1$.

Acknowledgements The authors would like to express their sincere gratitude to the anonymous referees for their comments and remarks, which improved the presentation of this paper.

\section{References}

[1] Bertz, S.H., Wright, W.F.: The graph theory approach to synthetic analysis: definition and application of molecular complexity and synthetic complexity. Graph Theory Notes N. Y. 35, 32-48 (1998)

[2] Buckly, F.: Mean distance of line graphs. Congr. Numer. 32, 153-162 (1981)

[3] Cohen, N., Dimitrov, D., Krakovski, R., S̆krekovski, R., Vukasinovič, V.: On Wiener index of graphs and their line graphs. MATCH Commun. Math. Comput. Chem. 64(3), 683-698 (2010)

[4] Deng, H.Y.: The Wiener index of a class of chemical graphs and their line graphs. J. Nat. Sci. Hunan Norm. Univ. 32(3), 23-26 (2009)

[5] Devillers, J., Balaban, A.T. (eds.): Topological Indices and Related Descriptors in QSAR and QSPR. Gordon and Breach, Reading (1999)

[6] Dobrynin, A.A., Entringer, R., Gutman, I.: Wiener index of trees: theory and applications. Acta Appl. Math. 66, 211-249 (2001)

[7] Dobrynin, A.A., Gutman, I., Jovašević, V.: Bicyclic graphs and its line graphs with the same Wiener index. Diskretn. Anal. Issled. Oper. Ser. 2 4(2), 3-9 (1997) (in Russian)

[8] Dobrynin, A.A., Gutman, I., Klavžar, S., Žigert, P.: Wiener index of hexagonal systems. Acta Appl. Math. 72, 247-294 (2002)

[9] Dobrynin, A.A., Mel'nikov, L.S.: Wiener index for graphs and their line graphs with arbitrary large cyclomatic numbers. Appl. Math. Lett. 18, 307-312 (2005)

[10] Dobrynin, A.A., Mel'nikov, L.S.: Wiener index, line graphs and the cyclomatic number. MATCH Commun. Math. Comput. Chem. 53, 209-214 (2005)

[11] Dobrynin, A.A., Mel'nikov, L.S.: Some results on the Wiener index of iterated line graphs. Electron. Notes Discrete Math. 22, 469-475 (2005) 
[12] Estrada, E., Guevara, N., Gutman, I.: Extension of edge connectivity index. Relationships to the line graph indices and QSPR applications. J. Chem. Inf. Comput. Sci. 38, 428-431 (1998)

[13] Gutman, I.: Distance of line graphs. Graph Theory Notes N. Y. 31, 49-52 (1996)

[14] Gutman, I., Estrada, E.: Topological indices based on the line graph of the molecular graph. J. Chem. Inf. Comput. Sci. 36, 541-543 (1996)

[15] Gutman, I., Pavlovič, L.: More on distance of line graphs. Graph Theory Notes N. Y. 33, 14-18 (1997)

[16] Nikolič, S., Trinajstič, N., Mihalič, Z.: The Wiener index: developments and applications. Croat. Chem. Acta 68, 105-129 (1995)

[17] Wiener, H.: Structural determination of paraffin boiling points. J. Am. Chem. Soc. 69, 17-20 (1947) 